\title{
Constant Stress Partially Accelerated Life Tests for Extended Generalized log Logistic Distribution Based on Type I Censored Competing Risks Data
}

\author{
Elgabry Gamalat, Rezk Hoda \\ Department of Statistics, Faculty of Commerce, AL-Azhar University, Cairo, Egypt \\ Email address: \\ dr.gamalatelgabry@yahoo.com (E. Gamalat), hodaragab2009@yahoo.com (R. Hoda) \\ To cite this article: \\ Elgabry Gamalat, Rezk Hoda. Constant Stress Partially Accelerated Life Tests for Extended Generalized log Logistic Distribution Based on \\ Type I Censored Competing Risks Data. Science Journal of Applied Mathematics and Statistics. Vol. 9, No. 1, 2021, pp. 15-19. \\ doi: 10.11648/j.sjams.20210901.12
}

Received: January 22, 2021; Accepted: February 7, 2021; Published: February 23, 2021

\begin{abstract}
As a result of technology improvement getting information about products and materials lifetimes under usual conditions. Therefore accelerated life testing or partially accelerated life testing usually are used to truncate the tests survives. The test items under accelerated life testing run under accelerated conditions and partially life tests run under both accelerated and use conditions. The main idea of accelerated life testing that the acceleration element is not unknown or the mathematical model relating the lifetime of the unit and the stress is known or can be assumed. In some cases, neither acceleration factor nor life-stress relations are not unknown. This paper concerned with studying and discussed the constant-stress partially accelerated life test (CPALT) under type I censored (T.I.C) competing risks data. Failure times resulting from T.I.C competing risks data are assumed to follow the Extended generalized log logistic (EGLL) distribution because this model is completely flexible to study positive data. This distribution is applied in various fields, for example lifetime studies, economics, finance and insurance. The maximum likelihood (ML) method is used to estimate the parameters under TIC competing risks data. The simulation algorithm is performed to assess the theoretical results of the maximum likelihood estimates based on TIC competing risks data.
\end{abstract}

Keywords: Constant Stress Partially Accelerated Life Test, Type I Censored Data, Cot Ending Failure Causes, Maximum Likelihood Method

\section{Introduction}

In experiments ALT is applied to reduce time and cast. There are different methods of acceleration. Among these methods, the constant stress in which stress on the units remains constant, the progressive stress in which the stress applied on the test units increases with time and the step stress in which the test conditions change for a given time or for a given number of failures, see DS. Bai, SW. [1]

In PALT the test items are run at both accelerated and normal conditions. PALT is suitable when the acceleration failure are the mathematical model is unknown, see AbdelHamid and Al-Hussaini [2], Hassan et.al.[3], Hassan et.al.[4], Abu-Zinadah and Ahmed [5], Ismail [6], A.A. Ismail, A.A. Albabtain [7], Ismail and Al Tamim [8], Ismail and Al Harbi [9], Li and Zheng [10], Zarrin, el al.[11], Fawzy [12],

The EGLL was first introduced by Lima and Corderio [13].
This new model is very flexible for modeling various types of data. In this paper, the MLES of the EGLL parameters are obtained under constant stress partially accelerated life test.

The rest of this paper is organized as follows: Competing risks schemes and model description are presented in Section 2. The ML estimators under T.I.C competing risks data is illustrated in Section 3. The simulation study is performed to assess the theoretical results in Section 4

\section{Competing Risks Plans and Model Description}

In Survival study, the items failure might be credit ready to more reason simultaneously. Theses "causes" would contending for the test unit failure. In the statistical literature, this issue is common as those contenting risks pattern. The information of competing risks study, the data comprises of a 
time failure and the related cause of its failure. It is assumed that the failure reasons are independent or reliant. In this study, we expect the suppressed failure time experiment, as proposed by Cox [14], that the times failure are individually circulated. where the failure is caused because of many reasons of failure, see Crowder [15].

Regarding experiment lifetime with i.i.d units $\mathrm{n}$ are drawn from $\mathrm{N}$ with i.i.d. Random variables (RVs) $Z_{1}, \ldots, Z_{n}$. Without loss of generality; accept that there are just two reasons of failure. We have

$$
T i=\min \left(Z_{1 i}, Z_{2 i}\right) \text { for } \mathrm{i}=1, \ldots, \mathrm{n},
$$

where $\mathrm{Z}_{1 i}, \mathrm{Z}_{2 i}$ indicates the underlying time failure of the $i-t h$ unit in $1^{\text {st }}$ and $2^{\text {nd }}$ failure reasons, separately. It is expected that the underlying time failure $\mathrm{Z}_{1 i}$ and $\mathrm{Z}_{2 i}$ are not dependent, the sets $\left(\mathrm{Z}_{1 i}, \mathrm{Z}_{2 i}\right)$ are i.i.d. The observed time failure is indicated through the $\mathrm{RV},=\min \left(Z_{1 i}, Z_{2 i}\right)$.

The reliability function (SF) of the RV T is defined as

$$
\bar{T}(z)=\mathrm{P}(T>z)=\mathrm{P}\left(T>z_{1}\right) \mathrm{P}\left(T>z_{2}\right)=\bar{F}(z) \bar{F}(z),
$$

Where $\bar{F}()=.1-F($.$) , and the SF is the reliability$ function of EGLL appropriation.

\section{Model Description and Its Assumptions}

In this section, we show the fundamental assumptions for the life test of the product in CPALT competing failure of the model. Additionally, the procedures of test in CS_PALT dependent on T.I.C plans that the competing failures lifetimes are expected to be EGLL distribution are clarified.
The procedure of test in CPALT is recognized as:

1) Whole $n$ units are split into two sets:

2) Group1(G.1) consists of $n_{1}=n(1-\pi),(1-\pi)$ is sample part units shared to typical circumstances.

3) Group 2 (G.2) consists of $n_{2}=n \pi$ residual units are exposed to accelerated conditions.

4) All items in G.1 and G.2 are run at steady stress level till the test ends after the time of censoring $\tau$ in the case of T.I.C is reached.

5) The lifetimes $T_{i}, i=1,2, \ldots, n(1-\pi)$, of units designated in ordinary states are EGLL distribution with shape parameter $\beta, b$, scale parameter $a$ and its probability density function (pdf) and cumulative distribution function (cdf) are given as follows:

$$
f\left(t_{i}\right)=\operatorname{\beta bat}_{i}^{b-1}\left(1+t_{i}^{b}\right)^{\beta-1} e^{-a\left[\left(1+t_{i}^{b}\right)^{\beta}-1\right]}, t_{i}, \beta, \alpha, b>0,
$$

and

$$
F\left(t_{i}\right)=1-e^{-a\left[\left(1+t_{i}^{b}\right)^{\beta}-1\right]},
$$

where the observed ordered failure times are $t_{(1)}<\cdots<$ $t_{(n u)}<\tau$ under T.I.C and $n u$ is the failed units number at ordinary situations.

6) The lifetimes $\left.X_{j}\right|_{(j=1,2, \ldots, n \pi)}$ of units assigned at accelerated conditions are a EGLL distribution with shape parameter $\beta, b$ and scale parameter a and its the pdf and cdf are given by:

$$
f\left(x_{j}\right)=\beta_{1} \beta b a\left(\beta_{1} x_{j}\right)^{b-1}\left(1+\left(\beta_{1} x_{j}\right)^{b}\right)^{\beta-1} e^{-a\left[\left(1+\left(\beta_{1} x_{j}\right)^{b}\right)^{\beta}-1\right]}, x_{j}, \beta, \alpha, b>0, \beta_{1}>1,
$$

And

$$
F\left(x_{j}\right)=1-e^{-a\left[\left(1+\left(\beta_{1} x_{j}\right)^{b}\right)^{\beta}-1\right]}
$$

where the observed ordered failure times are $x_{(1)}<\cdots<x_{(n a)}<\tau$ and na is the failed units number in accelerated situations under T.I.C.

Basic Assumption

1) The lifetimes $T_{i}, i=1,2, \ldots, n(1-\pi)$, of units approached at typical conditions are i.i.d RVs

2) The lifetimes $\left.X_{j}\right|_{(j=1,2, \ldots, n \pi)}$ of items designed at accelerated conditions are i.i.d RVs

3) The lifetimes $T_{i}$ and $X_{j}$ are commonly independent.

\section{ML Estimators Based on T.I.C Competing Risks Data}

Assume that the observed estimations of the whole lifetime $\mathrm{T}$ of size $n(1-\pi)$ at typical states are $t_{(1)}, t_{(2)}, \ldots, t_{(n(1-\pi))}$, and the observed number of the whole lifetime $X$ of size $n \pi$ at accelerated situation are $x_{(1)}, x_{(2)}, \ldots, x_{(n \pi)}$. Let $\delta_{u i}$ and $\delta_{a i}$ showed the indicators of the failure are

$$
\delta_{u i}=\mid \begin{gathered}
1, t_{i}>\tau \\
0, \text { otherwise }
\end{gathered} i=1,2, \ldots, n(1-\pi)
$$

and

$$
\delta_{a j}=\mid \begin{gathered}
1, x_{j}>\tau \\
0, \text { otherwise }
\end{gathered} i=1,2, \ldots, n \pi
$$


The probability work for T.I.C contending risks of data at when time the reason of failure is known at ordinary circumstances as follows:

$$
L \propto \prod_{i=1}^{n \bar{\pi}}\left[f_{1}\left(t_{i}\right) \bar{F}_{2}\left(t_{i}\right)\right]^{I\left(\delta_{i}=1\right)}\left[f_{2}\left(t_{i}\right) \bar{F}_{1}\left(t_{i}\right)\right]^{I\left(\delta_{i}=2\right)}\left[\bar{F}_{1}(\tau) \bar{F}_{2}(\tau)\right]^{\bar{\delta}_{i}},
$$

where, $t_{i}=t_{(i)}$, and $\bar{\pi}=(1-\pi)$. By substituting (1), (2), (3) and (4) in (5), then

$$
\begin{aligned}
& L_{1(u i)} \propto \prod_{i=1}^{n \bar{\pi}}\left[\beta_{1} b_{1} a_{1} t_{i}^{b_{1}-1}\left(1+t_{i}^{b_{1}}\right)^{\beta_{1}-1} e^{\left.-\left\{a_{1}\left[\left(1+t_{i} b_{1}\right)^{\beta_{1}}-1\right]+a_{2}\left[\left(1+t_{i} b_{2}\right)^{\beta_{2}}-1\right]\right\}\right]^{\delta_{1 u i}}\left[\beta_{2} b_{2} a_{2} t_{i}^{b_{2}-1}(1\right.}\right. \\
& \left.+t_{i}^{\left.b_{2}\right)^{\beta_{2}-1}} e^{-\left\{a_{1}\left[\left(1+t_{i}^{b_{1}}\right)^{\beta_{1}}-1\right]+a_{2}\left[\left(1+t_{i} b_{2}\right)^{\beta_{2}}-1\right]\right\}}\right]^{\delta_{2 u i}}\left[e^{\left.-\left\{a_{1}\left[\left(1+\tau^{b_{1}}\right)^{\beta_{1}}-1\right]+a_{2}\left[\left(1+\tau^{b_{2}}\right)^{\beta_{2}}-1\right]\right\}\right]^{\delta_{u i}}}\right.
\end{aligned}
$$

Additionally, the T.I.C. likelihood function contending risks information at what time the failure reason of failure is known at accelerated conditions is given by

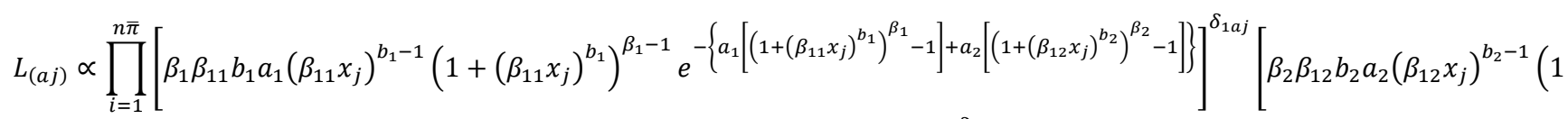

$$
\begin{aligned}
& \left.\left.+\left(\beta_{12} x_{j}\right)^{b_{2}}\right)^{\beta_{2}-1} e^{-\left\{a_{1}\left[\left(1+\left(\beta_{11} x_{j}\right)^{b_{1}}\right)^{\beta_{1}}-1\right]+a_{2}\left[\left(1+\left(\beta_{12} x_{j}\right)^{b_{2}}\right)^{\beta_{2}}-1\right]\right\}}\right]^{\delta_{2 a j}}\left[e^{\left.-\left\{a_{1}\left[\left(1+\left(\beta_{11} \tau\right)^{b_{1}}\right)^{\beta_{1}}-1\right]+a_{2}\left[\left(1+\left(\beta_{12} \tau\right)^{b_{2}}\right)^{\beta_{2}}-1\right]\right\}\right]^{\bar{\delta}_{a j}}}\right.
\end{aligned}
$$

Since the lifetimes of $t_{1}, \ldots, t_{n u}$ and $x_{1}, \ldots, x_{n a}$ are i.i.d then the whole T.I.C likelihood function competing risks data after the failure cause is known at typical and accelerated cases $\left(t_{1} ; \delta_{u 1} ; \ldots, t_{n \bar{\pi}} ; \delta_{u n \bar{\pi}} ; x_{1} ; \delta_{a 1} \ldots, x_{n \pi} ; \delta_{a n \pi}\right)$ is given by

$$
\begin{aligned}
& \prod_{i=1}^{n \bar{\pi}}\left[\beta_{1} b_{1} a_{1} t_{i}^{b_{1}-1}\left(1+t_{i}^{b_{1}}\right)^{\beta_{1}-1} e^{-\left\{a_{1}\left[\left(1+t_{i}^{b_{1}}\right)^{\beta_{1}}-1\right]+a_{2}\left[\left(1+t_{i} b_{2}\right)^{\beta_{2}}-1\right]\right\}}\right]^{\delta_{1 u i}}\left[L_{(u i)} L_{1(a j)} \propto\right.
\end{aligned}
$$

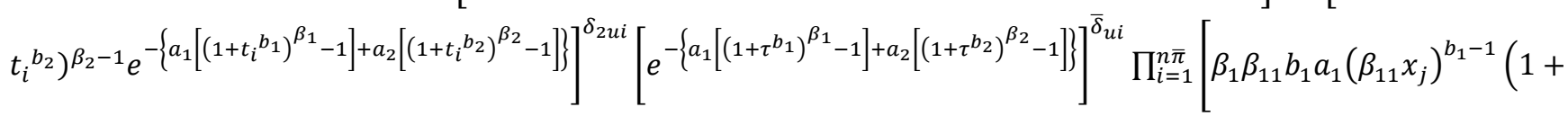

$$
\begin{aligned}
& \left.\left(\beta_{11} x_{j}\right)^{b_{1}}\right)^{\beta_{1}-1} e^{\left.-\left\{a_{1}\left[\left(1+\left(\beta_{11} x_{j}\right)^{b_{1}}\right)^{\beta_{1}}-1\right]+a_{2}\left[\left(1+\left(\beta_{12} x_{j}\right)^{b_{2}}\right)^{\beta_{2}}-1\right]\right\}\right]^{\delta_{1 a j}}}\left[\beta_{2} \beta_{12} b_{2} a_{2}\left(\beta_{12} x_{j}\right)^{b_{2}-1}(1+\right. \\
& \left.\left.\left(\beta_{12} x_{j}\right)^{b_{2}}\right)^{\beta_{2}-1} e^{-\left\{a_{1}\left[\left(1+\left(\beta_{11} x_{j}\right)^{b_{1}}\right)^{\beta_{1}}-1\right]+a_{2}\left[\left(1+\left(\beta_{12} x_{j}\right)^{b_{2}}\right)^{\beta_{2}}-1\right]\right\}}\right]^{\delta_{2 a j}}\left[e^{\left.-\left\{a_{1}\left[\left(1+\left(\beta_{11} \tau\right)^{b_{1}}\right)^{\beta_{1}}-1\right]+a_{2}\left[\left(1+\left(\beta_{12} \tau\right)^{b_{2}}\right)^{\beta_{2}}-1\right]\right\}\right]^{\delta_{a j}}}\right.
\end{aligned}
$$

where, $\bar{\delta}_{u i}=1-\delta_{u i}$ and $\bar{\delta}_{a j}=1-\delta_{a j}$.

The ML assessors $\hat{\beta}_{1}, \widehat{b}_{1}, \widehat{a}_{1}, \hat{\beta}_{2}, \widehat{b}_{2}, \widehat{a}_{2}, \hat{\beta}_{11}$ and $\hat{\beta}_{12}$ of the parameters and acceleration factors $\beta_{1}, b_{1}, a_{1}, \beta_{2}, b_{2}, a_{2}, \beta_{11}$ and $\beta_{12}$ are the values when boosts the likelihood function. The logarithm of the likelihood function $\ell=\ln L_{i}$ is given by:

$\ell \propto n \bar{\pi} \ln \beta_{1}+n \bar{\pi} \ln b_{1}+n \bar{\pi} \ln a_{1}+\left(b_{1}-1\right) \sum_{i=1}^{n \bar{\pi}} \ln \delta_{1 u i} t_{i}+\left(\beta_{1}-1\right) \sum_{i=1}^{n \bar{\pi}} \ln \delta_{1 u i}\left(1+t_{i}^{b_{1}}\right)-a_{1} \sum_{i=1}^{n \bar{\pi}} \delta_{1 u i}\left[\left(1+t_{i}^{b_{1}}\right)^{\beta_{1}}-\right.$ $1]-a_{2} \sum_{i=1}^{n \bar{\pi}} \delta_{1 u i}\left[\left(1+t_{i}^{b_{2}}\right)^{\beta_{2}}-1\right]+n \bar{\pi} \ln \beta_{2}+n \bar{\pi} \ln b_{2}+n \bar{\pi} \ln a_{2}+\left(b_{2}-1\right) \sum_{i=1}^{n \bar{\pi}} \ln \delta_{2 u i} t_{i}+\left(\beta_{2}-1\right) \sum_{i=1}^{n \bar{\pi}} \ln \delta_{2 u i}(1+$ $\left.t_{i}^{b_{2}}\right)-a_{2} \sum_{i=1}^{n \bar{\pi}} \delta_{2 u i}\left[\left(1+t_{i}^{b_{2}}\right)^{\beta_{2}}-1\right]-a_{1} \sum_{i=1}^{n \bar{\pi}} \delta_{2 u i}\left[\left(1+t_{i}{ }^{b_{1}}\right)^{\beta_{1}}-1\right]+n \pi \ln \beta_{1}+n \pi \ln b_{1}+n \pi \ln a_{1}+\left(b_{1}-\right.$ 1) $\sum_{j=1}^{n \pi} \ln \delta_{1 a j} x_{j}+\left(\beta_{1}-1\right) \sum_{j=1}^{n \bar{\pi}} \ln \delta_{1 a j}\left(1+x_{j}^{b_{1}}\right)-a_{1} \sum_{j=1}^{n \bar{\pi}} \delta_{1 a j}\left[\left(1+x_{j}^{b_{1}}\right)^{\beta_{1}}-1\right]-a_{2} \sum_{j=1}^{n \pi} \delta_{1 a j}\left[\left(1+x_{j}^{b_{2}}\right)^{\beta_{2}}-1\right]+$ $n \pi \ln \beta_{2}+n \pi \ln b_{2}+n \pi \ln a_{2}+\left(b_{2}-1\right) \sum_{j=1}^{n \bar{\pi}} \ln \delta_{2 a j} x_{j}+\left(\beta_{2}-1\right) \sum_{j=1}^{n \pi} \ln \delta_{2 a j}\left(1+x_{j} b_{2}\right)+-a_{2} \sum_{j=1}^{n \pi} \delta_{2 a j}[(1+$

$$
\left.\left.x_{j}^{b_{2}}\right)^{\beta_{2}}-1\right]-a_{1} \sum_{j=1}^{n \bar{\pi}} \delta_{2 a j}\left[\left(1+x_{j}^{b_{1}}\right)^{\beta_{1}}-1\right]-\bar{\delta}_{a j}\left\{a_{1}\left[\left(1+\left(\lambda_{k} \tau\right)^{b_{1}}\right)^{\beta_{1}}-1\right]+a_{2}\left[\left(1+\left(\lambda_{k} \tau\right)^{b_{2}}\right)^{\beta_{2}}-1\right]\right\}
$$

The first derivatives of the logarithm of the likelihood function (7) concerned to $\beta_{k}, b_{k}, a_{k}, \lambda_{k}, \mathrm{k}=1,2$, are given by

$$
\begin{gathered}
\frac{\partial \ell}{\partial \beta_{k}}=\frac{n \bar{\pi}}{\beta_{k}}+\sum_{i=1}^{n \bar{\pi}} \ln \delta_{k u i}\left(1+t_{i}^{b_{k}}\right)-a_{k} \sum_{j=1}^{n \pi} \delta_{k a j}\left[\left(1+x_{j}^{b_{k}}\right)^{\beta_{k}}\right] \ln \left(1+x_{j}^{b_{k}}\right)+\frac{n \pi}{\beta_{k}}+\sum_{i=1}^{n \pi} \ln \delta_{k u i}\left(1+t_{i}^{b_{k}}\right)-a_{k} \sum_{j=1}^{n \pi} \delta_{k a j}[(1+ \\
\left.\left.x_{j}^{b_{k}}\right)^{\beta_{k}}\right] \ln \left(1+x_{j}^{b_{k}}\right)-\bar{\delta}_{a j}\left\{a_{k}\left[\left(1+\left(\lambda_{k} \tau\right)^{b_{k}}\right)^{\beta_{k}}-1\right] \ln \left(1+\left(\lambda_{k} \tau\right)^{b_{k}}\right)\right\},
\end{gathered}
$$




$$
\begin{gathered}
\frac{\partial \ell}{\partial b_{k}}=\frac{n \bar{\pi}}{b_{k}}+\sum_{i=1}^{n \bar{\pi}} \ln \delta_{k u i} t_{i}+\left(\beta_{k}-1\right) \sum_{i=1}^{n \bar{\pi}} \ln \delta_{k u i}\left(1+t_{i}^{b_{k}}\right) \ln t_{i}-a_{k} \sum_{i=1}^{n \bar{\pi}} \delta_{k u i} \ln t_{i}\left[\left(1+t_{i}^{b_{k}}\right)^{\beta_{k}}-1\right] \\
\frac{n \pi}{b_{k}}+\sum_{i=1}^{n \bar{\pi}} \ln \delta_{k u i} t_{i}+\left(\beta_{k}-1\right) \sum_{i=1}^{n \bar{\pi}} \ln \delta_{k u i}\left(1+t_{i} b_{k}\right) \ln t_{i}-a_{k} \sum_{i=1}^{n \bar{\pi}} \delta_{k u i} \ln t_{i}\left[\left(1+t_{i}^{b_{k}}\right)^{\beta_{k}}-1\right]-\bar{\delta}_{a j}\left\{a_{k}\left[\left(1+\left(\lambda_{k} \tau\right)^{b_{k}}\right)^{\beta_{k}}-1\right]\right\}, \\
\frac{\partial \ell}{\partial a_{k}}=\frac{n \bar{\pi}}{a_{k}}+\sum_{i=1}^{n \bar{\pi}} \delta_{k u i}\left[\left(1+t_{i}^{b_{k}}\right)^{\beta_{k}}-1\right]+\frac{n \pi}{a_{k}}-\sum_{i=1}^{n \bar{\pi}} \delta_{k u i} \ln t_{i}\left[\left(1+t_{i}^{b_{k}}\right)^{\beta_{k}}-1\right]-\bar{\delta}_{a j}\left[\left(1+\left(\lambda_{k} \tau\right)^{b_{k}}\right)^{\beta_{k}}-1\right],
\end{gathered}
$$

and

$$
\frac{\partial \ell}{\partial \lambda_{k}}=\bar{\delta}_{a j} b_{k}\left\{a_{k}\left[\left(1+\left(\lambda_{k} \tau\right)^{b_{k}}\right)^{\beta_{k}}-1\right]\right\}\left(\lambda_{k} \tau\right)^{b_{k}-1} .
$$

Setting Eqs. (8)- (11) by zeros. The arrangement of these nonlinear conditions can't be solved analytically. Thus, numerical solution via iterative techniques is applied to obtain the ML estimators.

\section{Simulation Study}

A simulation study is used to assess the estimates performance. The assessments of the acceleration factor $\left(\lambda_{1}, \lambda_{2}\right)$ and population parameters $\beta_{1}, b_{1}, a_{1}, \beta_{2}, b_{2}, a_{2}$ are evaluated regarding the mean squared errors (MSEs) and biases. The numerical technique is approached as follows:

1) A random sample size $n_{1}=n(1-\pi)$, where $\pi=0.4$ is the ratio and $\mathrm{n}$ is the whole sample size, is generated under typical circumstances. So, samples are generated from $W_{1} \sim \operatorname{EGLL}\left(n_{1}, \beta_{1}, b_{1}, a_{1}\right)$ and $W_{2} \sim$ $\operatorname{EGLL}\left(n_{2}, \beta_{2}, b_{2}, a_{2}\right)$. Taking into account two generated samples generate new samples $t_{1}=$ $t_{(1)}, t_{(2)}, t_{(3)}, \ldots, t_{(n 1)}$ where

$$
T=\min \left(W_{1}, W_{2}\right) .
$$

2) A random sample size $n_{2}=n \pi$ is deduced under accelerated cases. Thus, the two samples from $W_{1} \sim$ $\operatorname{EGLL}\left(n_{1}, \beta_{1}, b_{1}, a_{1}\right)$ and $W_{2} \sim \operatorname{EGLL}\left(n_{2}, \beta_{2}, b_{2}, a_{2}\right)$ are generated. The two generated samples generate new samples

3) $X=x_{(1)}, x_{(2)}, x_{(3)}, \ldots, x_{(n 2)}$ where

$$
X=\min \left(W_{1}, W_{2}\right) \text {. }
$$

4) In T.I.C, let $\tau=1,1.5,2$ for sample sizes $30,60,100$, 200 and 500 , for some selected the unknown parameters and factor of accelerated, this process is iterated 1000 times at various estimations of population $\beta_{1}=$ $1.5, b_{1}=2, a_{1}=2.5, \beta_{2}=3, b_{2}=4, a_{2}=2 \quad, \quad \lambda_{1}=$ $1.5, \lambda_{2}=3$

5) The average estimations of biases (Abias) and MSEs are processed.

The mathematical results are reported in Table 1. The outcomes can be found as follows:

1) The Abias and MSEs decrease as $n$ increase under T.I.C data.

2) It is noticed that the Abias and MSEs decrease, censoring time $\tau$ decrease.

\begin{tabular}{|c|c|c|c|c|c|c|c|}
\hline \multirow{2}{*}{$\mathbf{n}$} & \multirow{2}{*}{ MLE } & \multicolumn{2}{|l|}{$\tau=2$} & \multicolumn{2}{|l|}{$\tau=1.5$} & \multicolumn{2}{|l|}{$\tau=1$} \\
\hline & & Abias & MSE & Abias & MSE & Abias & MSE \\
\hline \multirow{8}{*}{30} & $\hat{\beta}_{1}$ & 0.0923 & 0.0101 & 0.7760 & 0.0073 & 0.0456 & 0.0089 \\
\hline & $\hat{b}_{1}$ & 0.0853 & 0.0088 & 0.0706 & 0.0062 & 0.0686 & 0.0079 \\
\hline & $\hat{a}_{1}$ & 0.1894 & 0.0375 & 0.1747 & 0.0315 & 0.1427 & 0.0330 \\
\hline & $\hat{\lambda}_{1}$ & 0.1174 & 0.0154 & 0.1027 & 0.0116 & 0.1005 & 0.0134 \\
\hline & $\hat{\beta}_{2}$ & 0.1836 & 0.0353 & 0.1689 & 0.0298 & 0.1669 & 0.0310 \\
\hline & $\hat{b}_{2}$ & 0.1642 & 0.0286 & 0.1495 & 0.0236 & 0.1475 & 0.0250 \\
\hline & $\hat{a}_{2}$ & 0.1507 & 0.0243 & 0.1360 & 0.0193 & 0.1340 & 0.0212 \\
\hline & $\hat{\lambda}_{2}$ & 0.1054 & 0.0127 & 0.0907 & 0.0005 & 0.0887 & 0.0111 \\
\hline \multirow{8}{*}{60} & $\hat{\beta}_{1}$ & 0.0462 & 0.0037 & 0.0315 & 0.0022 & 0.0295 & 0.0022 \\
\hline & $\hat{b}_{1}$ & 0.0426 & 0.0034 & 0.0279 & 0.0020 & 0.0258 & 0.0019 \\
\hline & $\hat{a}_{1}$ & 0.0532 & 0.0044 & 0.0385 & 0.0027 & 0.0365 & 0.0025 \\
\hline & $\hat{\lambda}_{1}$ & 0.0476 & 0.0038 & 0.0329 & 0.0023 & 0.0309 & 0.0021 \\
\hline & $\hat{\beta}_{2}$ & 0.0612 & 0.0053 & 0.0465 & 0.0034 & 0.0445 & 0.0020 \\
\hline & $\hat{b}_{2}$ & 0.0547 & 0.0046 & 0.0400 & 0.0028 & 0.0380 & 0.0027 \\
\hline & $\hat{a}_{2}$ & 0.0754 & 0.0073 & 0.0607 & 0.0049 & 0.0587 & 0.0036 \\
\hline & $\hat{\lambda}_{2}$ & 0.0624 & 0.0034 & 0.0270 & 0.0019 & 0.0250 & 0.0017 \\
\hline \multirow{8}{*}{100} & $\hat{\beta}_{1}$ & 0.0131 & 0.0018 & 0.0016 & 0.0012 & 0.0036 & 0.0032 \\
\hline & $\hat{b}_{1}$ & 0.0135 & 0.0019 & 0.0012 & 0.0013 & 0.0032 & 0.0023 \\
\hline & $\hat{a}_{1}$ & 0.0163 & 0.0019 & 0.0015 & 0.0012 & 0.0004 & 0.0032 \\
\hline & $\hat{\lambda}_{1}$ & 0.0104 & 0.0017 & 0.0043 & 0.0013 & 0.0063 & 0.0025 \\
\hline & $\hat{\beta}_{2}$ & 0.0046 & 0.0016 & 0.0101 & 0.0013 & 0.0121 & 0.0034 \\
\hline & $\hat{b}_{2}$ & 0.0099 & 0.0016 & 0.0048 & 0.00125 & 0.0068 & 0.0033 \\
\hline & $\hat{a}_{2}$ & 0.0165 & 0.0019 & 0.0018 & 0.00135 & 0.0021 & 0.0031 \\
\hline & $\hat{\lambda}_{2}$ & 0.0111 & 0.0017 & 0.0036 & 0.00124 & 0.0056 & 0.0034 \\
\hline
\end{tabular}

3) Clearly, the acceleration of the experiment is useful to get outcomes and data quickly, yet the most consequences of normal condition are more exact that speeding up condition.

Table 1. Abias and MSEs of MLE based on T.I.C at $\tau=1,1.5,2$ and $\pi=0.4$. 


\begin{tabular}{|c|c|c|c|c|c|c|c|}
\hline \multirow{2}{*}{ n } & \multirow{2}{*}{ MLE } & \multicolumn{2}{|l|}{$\tau=2$} & \multicolumn{2}{|l|}{$\tau=1.5$} & \multicolumn{2}{|l|}{$\tau=1$} \\
\hline & & Abias & MSE & Abias & MSE & Abias & MSE \\
\hline \multirow{8}{*}{200} & $\hat{\beta}_{1}$ & 0.0065 & 0.00004 & 0.0052 & $2.74 \mathrm{x} \mathrm{e}^{-5}$ & 0.00393 & $1.54 \mathrm{x} \mathrm{e}^{-5}$ \\
\hline & $\hat{b}_{1}$ & 0.0079 & $6.65 \times \mathrm{x}^{-5}$ & 0.0064 & $2.92 \mathrm{x} \mathrm{e}^{-5}$ & 0.0041 & $1.64 \mathrm{x} \mathrm{e}^{-5}$ \\
\hline & $\hat{a}_{1}$ & 0.0081 & $6.64 \times \mathrm{e}^{-5}$ & 0.0065 & $4.25 \mathrm{x} \mathrm{e}^{-5}$ & 0.0049 & $2.39 \times \mathrm{xe}^{-5}$ \\
\hline & $\hat{\lambda}_{1}$ & 0.0052 & $2.7 \times \mathrm{e}^{-5}$ & 0.0042 & $1.73 \mathrm{x} \mathrm{e}^{-5}$ & 0.0031 & $9.37 \times \mathrm{e}^{-6}$ \\
\hline & $\hat{\beta}_{2}^{1}$ & 0.0023 & $5 \times \mathrm{e}^{-6}$ & 0.0018 & $3.38 \times \mathrm{x} \mathrm{e}^{-6}$ & 0.0014 & $1.90 \times \mathrm{x} \mathrm{e}^{-6}$ \\
\hline & $\hat{b}_{2}$ & 0.0049 & $2.45 \mathrm{x} \mathrm{e}^{-5}$ & 0.0040 & $1.57 \mathrm{x} \mathrm{e}^{-5}$ & 0.0030 & $8.82 \times \mathrm{e}^{-6}$ \\
\hline & $\hat{a}_{2}$ & 0.0083 & $6.80 \times \mathrm{x}^{-5}$ & 0.0066 & $4.36 \times \mathrm{x}^{-5}$ & 0.0049 & $2.45 \times \mathrm{e}^{-5}$ \\
\hline & $\hat{\lambda}_{2}$ & 0.0055 & $3.08 \times \mathrm{x}^{-5}$ & 0.0044 & $1.97 \mathrm{x} \mathrm{e}^{-5}$ & 0.0033 & $1.11 \mathrm{x} \mathrm{e}^{-5}$ \\
\hline
\end{tabular}

Table 1. Continued.

\begin{tabular}{|c|c|c|c|c|c|c|c|}
\hline \multirow{2}{*}{ n } & \multirow{2}{*}{ MLE } & \multicolumn{2}{|l|}{ Abias } & \multicolumn{2}{|l|}{ MSE } & \multicolumn{2}{|l|}{ Abias } \\
\hline & & Abias & MSE & Abias & MSE & Abias & MSE \\
\hline \multirow{8}{*}{500} & $\hat{\beta}_{1}$ & 0.0016 & $2.64 \times \mathrm{x}^{-6}$ & 0.00032 & $1.54 \times \mathrm{x}^{-6}$ & 0.00028 & $8.09 \times \mathrm{x} \mathrm{e}^{-8}$ \\
\hline & $\hat{b}_{1}$ & 0.0019 & $3.90 \times \mathrm{e}^{-6}$ & 0.00039 & $3.15 \mathrm{x} \mathrm{e}^{-6}$ & $3.56 \times \mathrm{e}^{-4}$ & $1.17 \mathrm{x} \mathrm{e}^{-7}$ \\
\hline & $\hat{a}_{1}$ & 0.0020 & $4.10 \times \mathrm{e}^{-6}$ & 0.00040 & $3.83 \mathrm{x} \mathrm{e}^{-6}$ & $2.49 \times \mathrm{x} \mathrm{e}^{-4}$ & $1.26 \mathrm{x} \mathrm{e}^{-7}$ \\
\hline & $\hat{\lambda}_{1}$ & 0.0013 & $1.69 \mathrm{x} \mathrm{e}^{-6}$ & 0.00026 & $1.21 \mathrm{x} \mathrm{e}^{-6}$ & 0.00227 & $5.18 \times \mathrm{e}^{-8}$ \\
\hline & $\hat{\beta}_{2}$ & 0.0006 & $3.31 \mathrm{x} \mathrm{e}^{-7}$ & 0.00012 & $2.27 \mathrm{x} \mathrm{e}^{-7}$ & $1.01 \mathrm{x} \mathrm{e}^{-4}$ & $1.16 \mathrm{x} \mathrm{e}^{-8}$ \\
\hline & $\hat{b}_{2}$ & 0.0013 & $1.50 \times \mathrm{e}^{-6}$ & 0.00025 & $1.51 \mathrm{x} \mathrm{e}^{-6}$ & $2.05 \mathrm{x} \mathrm{e}^{-4}$ & $4.60 \times \mathrm{e}^{-8}$ \\
\hline & $\hat{a}_{2}$ & 0.0021 & $4.31 \times \mathrm{e}^{-6}$ & 0.00045 & $3.73 \times \mathrm{xe}^{-6}$ & $3.13 \mathrm{x} \mathrm{e}^{-4}$ & $1.32 \mathrm{x} \mathrm{e}^{-7}$ \\
\hline & $\hat{\lambda}_{2}$ & 0.0014 & $1.89 \mathrm{x} \mathrm{e}^{-6}$ & 0.00025 & $1.89 \mathrm{x} \mathrm{e}^{-6}$ & $2.41 \mathrm{x} \mathrm{e}^{-5}$ & $5.79 \times \mathrm{x}^{-8}$ \\
\hline
\end{tabular}

\section{Conclusion}

In this study, the CPALT under T.I.C data is discussed assuming that failure times are EGLL distribution. The ML method is applied to estimate the unknown parameters under TIC competing risks. To assess the theoretical results of the ML method for CPALT based on T.I.C data, the simulation algorithm is performed. It is noted that the Abias and MSEs decrease as $\mathrm{n}$ increase under T.I.C data and censoring time $\tau$ decrease. The acceleration of the experiment is valuable helpful to get outcomes and data quickly, yet the most consequences of normal condition are more exact that speeding up condition.

\section{Conflicts of Interest}

The authors declare no conflict of interest.

\section{References}

[1] DS. Bai, SW. Chung, Chun YR Optimal design of partially accelerated life tests for the lognormal distribution under type I censoring. Reliab Eng Syst Saf 40 (1) (1993): 85-92.

[2] A. Abdel-Hamid, E K. Al-Hussaini. Inference and Optimal Design Based on Step-Partially Accelerated Life Tests for the Generalized Pareto Distribution under Progressive Type-I Censoring, Communications in Statistics - Simulation and Computation, 44, (2014); 1750-1769.

[3] AS. Hassan, MS. Assar, AN, Zaky Constant-stress partially accelerated life tests for inverted Weibull distribution with multiple censored data. Int J Adv Stat Probab 3 (1) (2015): 72-82.

[4] A S. Hassan, S. G. Nassr, S. Pramanik, S. S. Maiti. Estimation in Constant Stress Partially Accelerated Life Tests for Weibull Distribution Based on Censored Competing Risks Data, Annals of Data Science, 7 (7) (2019): 45-62.

[5] H H. Abu-Zinadah and N. S. Ahmed Competing Risks Model with Partially Step-Stress Accelerate Life Tests in Analyses Lifetime Chen Data under Type-II Censoring Scheme, Open Physics, 7 (1) (2019): 192-199.

[6] A. A. Ismail. Inference in the generalized exponential distribution under partially accelerated tests with progressive Type-II censoring", Theoretical and Applied Fracture Mechanics, 59 (2012): 49-56.

[7] A.A. Ismail, A.A. Al-babtain, Planning failure-censored constant-stress partially accelerated life test, Journal of Systems Engineering and Electronics, 26 (3) (2015): 644-650.

[8] Ali A. Ismail, A. Al Tamimi. Optimum Constant- Stress Partially Accelerated Life Test Plans Using Type-I Censored Data from the Inverse Weibull Distribution, Strength of Materials, 9 (3), (2018): 1-9.

[9] A. A. Ismail, M. M. Al-Harbi, Statistical Inference of Constant-Stress Partially Accelerated Life Test Model Using Failure-Censored Data from the Linear Failure Rate Distribution, Strength of Materials, 51, (2019): 122-129.

[10] X. Li, H. Zheng, Estimation and Optimum Constant-Stress Partially Accelerated Life Test Plans for Gompertz Distribution with Type with Type-I Censoring, Communications in Statistics - Theory and Methods (2015): 1-15.

[11] S. Zarrin, M. Kamal, S. Saxena, Estimation constant stress partially accelerated life tests for Rayleing distribution under Type-I Censoring, Reliability Theory and Applications 4 (27) $201241-52$.

[12] Mohamad A. Fawzy. Prediction of Kumaraswamy distribution in constant - stress model based on type - I hybrid censored data, Statistical Analysis and Data Mining: The ASA Data Science Journal, 13 (2020): 205-215.

[13] S. Lima, and G. Corderio, The Extended Log-Logistic Distribution: Properties and Application, Annals of the Brazilian Academy of Sciences, 89 (2017), 3-17.

[14] DR. Cox The analysis of exponentially distributed with two types of failure. J R Stat Ser B 21 (1959): 411-421.

[15] MJ. Crowder Classical competing risks model. Chapman \& Hall/CRC, (2001) New York. 